\title{
Toward classification criteria for early osteoarthritis of the knee
}

\author{
F.P. Luyten ${ }^{\mathrm{a}, *}$, S. Bierma-Zeinstra ${ }^{\mathrm{b}, \mathrm{c}}$, F. Dell'Accio ${ }^{\mathrm{d}}$, V.B. Kraus ${ }^{\mathrm{e}}$, K. Nakata ${ }^{\mathrm{f}}$, I. Sekiya ${ }^{\mathrm{g}}$, \\ N.K. Arden ${ }^{\text {h,1 }}$, L.S. Lohmander ${ }^{\mathrm{i}, 1}$ \\ a Department of Development and Regeneration, Skeletal Biology and Engineering Research Center, KU Leuven, Herestraat 49 Box 813, B-3000 Leuven, \\ Belgium \\ b Department of General Practice, University Medical Center Rotterdam, Erasmus MC, Rotterdam, the Netherlands \\ ${ }^{\mathrm{c}}$ Department of Orthopedics, University Medical Center Rotterdam, Erasmus MC, Rotterdam, the Netherlands \\ ${ }^{\mathrm{d}}$ William Harvey Research Institute, Barts and the London School of Medicine and Dentistry, Queen Mary University of London, London, UK \\ e Division of Rheumatology, Duke Molecular Physiology Institute, Duke University School of Medicine, Durham, NC \\ ${ }^{\mathrm{f}}$ Medicine for Sports and Performing Arts, Department of Health and Sport Sciences, Osaka University Graduate School of Medicine, Osaka, Japan \\ ${ }^{g}$ Center for Stem Cell and Regenerative Medicine, Tokyo Medical and Dental University, Tokyo, Japan \\ ${ }^{\mathrm{h}}$ Arthritis Research UK Centre for Sport, Exercise and Osteoarthritis, University of Oxford, Oxford, England \\ i Department of Clinical Sciences, Faculty of Medicine, Lund University, Lund, Sweden
}

\section{A R T I C L E I N F O}

Keywords:

Early osteoarthritis

Knee

Classification criteria

\begin{abstract}
A B S T R A C T
Objective: To propose draft classification criteria for early stage osteoarthritis (OA) of the knee for use in a primary care setting.

Methods: A group of basic scientists, physician-scientists, rheumatologists, orthopedic surgeons, and physiotherapists in a workshop setting discussed potential classification criteria for early osteoarthritis of the knee. The workshop was divided into sessions around relevant topics with short state of the art presentations followed by breakout sessions, consensus discussions, and consolidation into a consensus document.

Results: Three classes of criteria were agreed: (1) Pain, symptoms/signs, self-reported function, and quality of life using tools such as KOOS: scoring $\leq 85 \%$ in at least 2 out of these 4 categories; (2) Clinical examination: at least 1 present out of joint line tenderness or crepitus; (3) Knee radiographs: Kellgren \& Lawrence $(\mathrm{KL})$ grade of 0 or 1 . MRI is at present not recommended as an aid to identify or define early OA in routine clinical practice or primary care, in light of the absence of validated consensus criteria and the high population prevalence of structural joint changes detected by this method. Biomarkers may have future utility in early OA classification, but no individual or set of biomarkers is yet robust enough. Conclusion: Based on our consensus proposal, draft classification criteria for early OA of the knee for use in clinical studies should include patient reported outcomes such as pain and function, together with clinical signs and KL grade $0-1$ on radiographs.
\end{abstract}

(c) 2017 Elsevier Inc. All rights reserved.
Knee osteoarthritis (OA) is the most common joint disease and is associated with significant pain, disability, premature mortality [1], and costs estimated around $1.5-2 \%$ of the GDP in western countries [2]. It is a complex, multifactorial disease with many different phenotypes [3]. In part due to this complexity and phenotypic heterogeneity, there are no proven disease modifying drugs approved by the regulatory agencies. However, new treatment targets are being identified, raising hopes for new treatments affecting disease progression [4].

The provision of time and healthcare resources are currently focused on the treatment of established OA (tertiary prevention), defined by chronic pain and manifest radiographic signs of

\footnotetext{
* Corresponding author.

E-mail address: frank.luyten@uzleuven.be (F.P. Luyten).

1 Co-senior authorship.
}

osteoarthritis. Classification criteria [5] and clinical guidance [6,7] have been issued for such patients with established and advanced joint damage. However, patients that fulfill these criteria commonly have significant joint damage and resultant altered biomechanics, and often altered pain processing due to the chronic pain. Effective treatment is therefore difficult. We regard this current mode of managing OA as being 'reactive', with treatment modalities added as symptoms worsen and the disease progresses toward an end stage, often, but not invariably, requiring joint replacement. This 'reactive' management mode of a common, costly, and severe disease can be compared with the now takenfor-granted 'proactive' emphasis on early diagnosis, management, and prevention of other common conditions like diabetes, cardiovascular disease, Alzheimer's disease, rheumatoid arthritis, osteoporosis, and cancer. To change our current reactive approach of managing $\mathrm{OA}$ into a proactive one, with an emphasis on secondary 
prevention, we need to identify risk factors and diagnose OA early, for a greater chance to prevent or slow disease progression.

We propose that treatment will be most effective in early disease. Unfortunately, despite the availability of diagnostic and classification criteria for established OA [8], the lack of a consensus on what represents early knee OA severely limits the feasibility of clinical trials. The Fibroblast Growth Factor 18 trial provides an illustrative example, where patients had severe OA of the medial femoral condyle and milder disease in the lateral compartment. Analysis of secondary outcomes revealed no change in cartilage loss in the medial compartment with severe OA, but a dosedependent statistically significant reduction of cartilage loss in the lateral compartment, characterized by milder OA [9]. Defining 'early $\mathrm{OA}^{\prime}$ would allow testing of an intervention in a patient population that is more likely to benefit from the intervention. Identifying $\mathrm{OA}$ in its earliest stages in the primary care setting would also allow the optimal multimodal management of the disease with, e.g., patient education and exercise [10,11]. Proactive modification of the course of disease, before destructive processes irreversibly compromise the joint, could prevent development of chronic pain before it elicits sensitization locally and centrally, and before functional impairment leads to deconditioning. Such early intervention could limit indirect costs of the disease caused by loss of work participation, sick leave, lost productivity, premature retirement, and lifetime disease burden. In most countries, we would identify and manage these patients in primary care. To achieve this aim, we need to be able to identify early OA by signs and symptoms, joint structure changes, or soluble biomarkers. We also need tools to measure its progression and response to intervention in clinical studies.

However, we lack consensus criteria for classifying early OA. Importantly, classification criteria are standardized definitions primarily intended to create homogeneous patient subsets, recruited from first or second line caregivers, for the purpose of clinical studies $[12,13]$. The First International Early Knee Osteoarthritis (IEKO) workshop, held in Tokyo, Japan, in November 2014, and hosted by the Japanese Society for Early Osteoarthritis, gathered an international group of about 85 experts and participants (list see below in acknowledgments) to develop a first draft of classification criteria for early knee osteoarthritis. To add to its clinical relevance, a second independent, international group has produced a core set of outcome criteria for early OA (Emery et al., submitted).

\section{Methods}

The invited experts were selected based on their internationally recognized area expertise, experience, and availability to discuss a first draft of classification criteria for early knee osteoarthritis. Participants were selected to represent the following areas: rheumatology, orthopedic surgery, radiology, physiotherapy, epidemiology, health sciences, experimental research, basic and translational science related to OA. A list of workshop participants is provided in acknowledgments.

It was agreed at the outset of the meeting that the starting point ('base case') for the discussions would be the patient presenting with incident knee symptoms and/or signs in a primary care setting, with no clear history of recent trauma.

The workshop was divided into sessions around topics relevant for classification of the early OA patient, with short state-of-the-art presentations, followed by breakout sessions where specific questions were discussed in 10 smaller parallel breakout groups of 6-9 participants. A selection of questions was discussed and compiled before the meeting between the scientific coordinators (F.P.L. and L.S.L.) and the session chairpersons. Sessions were moderated by facilitators and group discussions by rapporteurs. Discussed topics included: general concepts of early and established OA; symptoms, function, and signs; structural assessment by X-ray imaging, MRI, arthroscopy, or ultrasound; molecular markers; and the role of inflammation and biomechanics. Following the breakout discussions, the rapporteurs presented the consensus answers of each group to the questions, followed by a general discussion. The facilitator concluded each session with a résumé of the consensus discussion, approved by the participants.

In a final session, the chairs of the workshop presented an overall summary based on previous session summaries, followed by a general discussion with any summary modifications and final version approved by participant consensus. With F.P.L. and L.S.L. as chairs, rapporteurs and co-rapporteurs merged the session summaries into a first draft.

\section{Results}

Symptoms, signs, and function

Participants agreed that symptoms and signs may be present at the earliest stages of the disease and therefore have potential for classification of early OA. To be useful criteria, participants further agreed that knee symptoms and signs should precede a diagnosis of established OA, defined as knee symptoms and Kellgren \& Lawrence $(\mathrm{KL})$ grade 2 or greater $(\mathrm{KL} \geq 2)$ radiographic changes $[14,15]$.

As summarized during the session, several observational studies have explored knee pain and other symptoms and signs [16-21], or symptoms and preradiographic joint changes on magnetic resonance imaging (MRI) [22-25] as predictors of future radiographic knee OA. These studies have shown that preradiographic joint changes detectable on MRI may predict incident radiographic knee OA by several years. However, as discussed in the imaging section of this report, MRI shows lesions in the tibiofemoral joint in most middle-aged and older people with no evidence of radiographic OA, regardless of pain [26,27]. This suggests that much further work will be needed to clarify associations between knee symptoms and MRI features.

Importantly, incident knee pain in the adult, in particular when using stairs, may predict incident radiographic knee OA by 2-3 years [28]. Isolated patellofemoral OA is often present in those with knee pain and predates tibiofemoral OA; signs and symptoms associated with isolated patellofemoral OA are crepitus and difficulty with descending stairs [29]. Joint line tenderness predicts the progression to established osteoarthritis in cohorts of patients with knee pain $[30,31]$.

In a cohort of early and established knee OA, the early OA group reported pain and symptoms assessed by KOOS domains that were comparable with the established OA group [32]. Loss of function may also be a relevant criterion to mark incident early OA. As an example, in a cohort study comparing healthy controls to patients with early knee OA defined primarily by MRI [33], and patients with established knee OA, the early OA group had significantly worse KOOS pain and ADL score compared to the control group [34]. Of note, isometric and isokinetic quadriceps strengths were lower, and varus thrust increased in the early OA patients, while other functional measures were not different [35].

Finally, a systematic review of prognostic factors for progression of clinical osteoarthritis of the knee found strong evidence for multiple prognostic factors but the large variety in definitions of clinical knee OA and disease progression hindered meta-analyses. Authors noted that evidence for the majority of determinants was limited or conflicting and that only few studies had used pain progression as an outcome [36]. 


\section{Breakout session}

During the round table discussions, four issues relevant to the development of classification criteria for early OA were discussed:

A) Should pain be included in the classification criteria? How would we describe this in terms of duration of pain and intensity?

Consensus was reached that pain should be included in the classification criteria and that ideally duration, severity and/or frequency should be specified. Consensus opinion considered the KOOS score the most appropriate currently available tool, as it includes all the WOMAC 3.0 questions, encompasses multiple domains of function, has minimal (10\%) ceiling effect (higher score indicates less impairment), and no floor effect [37]. This would make it applicable to the traditional older OA patient, but also particularly useful in younger people for whom evaluation for early OA would be relevant. The duration of symptoms was also discussed during the breakout session. The general consensus was that in early stages the pain comes and goes, and that there should have been repeated pain periods; the duration and frequency of these periods were discussed but not further defined based on lack of evidence. Previously, pain on most days of any 1 month of the last year was proposed [38].

Compartmental involvement, patellofemoral or tibiofemoral, and how this is presenting in early OA, was not discussed at the meeting. Future studies should further explore this parameter in the early OA population.

B) Should we categorize the nature of the pain as, e.g., inflammatory vs mechanical, allodynia, and peripheral/central pain?

The groups agreed that while insufficient data are available to link the diagnosis of early knee OA to one or more specific pain patterns, the consensus recommendation was that specific concomitant pain syndromes such as fibromyalgia should be excluded from clinical studies in early knee OA.

C) Should other symptoms and signs such as discomfort, locking, crepitus, or instability be included?

There was no overall consensus reached, in that four groups felt that crepitus should be included, three for joint line tenderness, three for stiffness, and two for swelling. Discomfort, locking, and instability were not thought to be useful in early OA.

D) Should function be included in the classification criteria, and if yes, how should it be defined and measured?

Due to the challenges of performing objective functional tests in primary care, consensus was that subjective function scores were the most appropriate [39], although simple tests such as walking speed, chair rise, and simple muscle strength tests could be useful. With the swift advent of mobile 'wearables' to measure functioning in daily life with ever increasing precision, it may soon be possible to include these types of assessments as part of the classification criteria, although they may be more suited as outcomes.

\section{Consensus statement}

Pain and self-reported function should be measured using validated tools such as KOOS. Signs, such as joint line tenderness, effusion, and crepitus may also be appropriate for use in the classification criteria of early OA.

\section{$X$-rays, ultrasound, and arthroscopy}

Participants agreed that ultrasound and arthroscopy, although used for visualizing joint structures in some settings, were not generally useful in the setting of primary care. The discussion focused on the utility of plain radiographs in the setting of early OA. While radiographic examination may not always be needed to diagnose knee $\mathrm{OA}$ in the primary care setting, evidence was presented in the session that even doubtful features of OA, such as minor osteophytes, add considerably to the predictive value of age, gender, BMI and 'questionnaire-based' factors, biomarkers, or a genetic risk score for subsequent OA development [40]. Participants agreed that validation of classification criteria for early OA would be accomplished by demonstrating high positive predictive value of the criteria for subsequent incident symptomatic, radiographic OA.

\section{Breakout session}

Should knee radiographs form part of the classification criteria? The consensus position of the participants was that for several reasons radiographs should be included. Firstly, without a knee radiograph, it would be difficult to rule out established OA, as symptoms and signs of early and established OA may overlap. Secondly, in the clinical setting, a radiograph would help rule out differential diagnoses such as stress fractures and osteonecrosis. The final reason was that there was recent evidence that changes in bone shape can be detected early in the course of knee OA and that in the future, this may provide a useful predictor or criterion for classification [41].

Which type of radiographic examination should be performed and how should it be assessed? Consensus was reached that two views would need to be taken to address the tibio-femoral (TF) and patella-femoral (PF) joints. To assess the TF joint, a fixed flexion Posterior-Anterior weight-bearing radiograph should be used with a standardized acquisition protocol, including placement of a measurement tool (sphere or ruler) on the film. The skyline view (supine), following a standardized protocol was the preferred view to assess the PF joint. Radiograph assessment would normally be performed according to local clinical policies, however the group did discuss the optimum method for assessing radiographs for research purposes, as this would require research to validate the new criteria. Assessment should be performed using wellvalidated semi-quantitative atlases. However, there was considerable discussion about the choice of atlas. The limitations of the KL atlas were discussed, particularly the scoring of grade 2 and its variability across different centers and cohorts. In the absence of obvious alternatives with significantly better performance, it was agreed that a KL grade of 0 or 1 should be used in the classification criteria. The scoring of the patellofemoral joint was less certain as it was not scored as part of the original KL criteria; however, several alternatives, such as the OARSI scoring system (with scores reflecting a definite osteophyte and/or joint space narrowing) do exist [42], and would need to be explored.

\section{Consensus statement}

There was a unanimous recommendation that radiographs should be used and that a KL grade of 0 or 1 should apply for early OA. Alignment with OARSI recommendations with regard to image acquisition and assessment was advised [43].

\section{Magnetic resonance imaging}

Magnetic resonance imaging (MRI) provides a highly sensitive, but not necessarily specific method to detect structural joint changes These changes can be assessed qualitatively, semi-quantitatively, or 
quantitatively by semi-automated or fully automated methods. MRI can also be used to assess tissue properties using compositional techniques. MRI is resource consuming, expensive, and usually not available in routine primary care. However, for research and validation of early OA classification criteria, MRI will be an important tool.

All speakers emphasized the sensitivity of MRI to detect joint tissue changes associated with osteoarthritis, but also that structural changes commonly associated with osteoarthritis are very common in the population, irrespective of joint symptoms, and increase in prevalence with age. For example, it has been reported that meniscal tears were present in $15 \%$ of women and $30 \%$ of men in an unselected population between ages 50 and 59, regardless of knee pain [44]. The prevalence increases with age. Cartilage damage, osteophytes, bone marrow lesions, and meniscal tears were very commonly present in knees of persons aged between 50 and 90 years without signs of osteoarthritis on routine knee radiography, again regardless of knee pain $[27,45]$. Therefore, a major challenge lies in the common occurrence of these changes in the general population, their modest cross-sectional association with joint symptoms, and their insufficiently documented natural history.

A draft MRI-based definition of tibiofemoral OA was suggested to include the presence of both group $[A]$ features, or one group [A] feature and two or more group [B] features [33,46]: Group [A] features include (i) definite osteophyte formation and (ii) full thickness cartilage loss; Group [B] features include (i) subchondral bone marrow lesion or cyst not associated with meniscal or ligamentous attachments, (ii) meniscal subluxation, maceration, or degenerative horizontal tear, (iii) partial thickness cartilage loss where full thickness loss is not present, and (iv) bone attrition [47].

This tentative definition will need validation in independent study populations. Relevant to this challenge, a recent study presented evidence to suggest that MRI-detected knee joint lesions in a population at-risk for osteoarthritis (but without plain radiographic signs of $\mathrm{OA}$ ) are not incidental and may represent early disease in persons at increased risk of definite knee OA [22]. In support, a 'hidden osteophyte', undetectable on plain X-ray but detectable on MRI, was predictive of development of knee osteoarthritis within 48 months [48]. Finally, synovitis in OA detected by MRI is associated with knee pain and can precede, by at least a year, incident radiographic OA [49].

\section{Consensus statement}

There was general agreement that MRI is a powerful technique that is needed in research on early OA. However, at present MRI is not recommended as an aid to identify or define early OA in routine clinical practice or primary care, in light of lack of validated consensus criteria, and the high population prevalence of structural joint changes detected by this method.

\section{Biomarkers}

The OA disease process represents a continuum from the earliest changes in cells through molecular changes in the surrounding matrix, having functional and structural consequences on the nano and micro scale, to emerging symptoms and structural changes detectable by imaging, and to the eventual joint destruction in some individuals. As our techniques to detect changes earlier in the disease process become more sensitive, we may be able detect OA earlier in the process.

Biomarkers of early OA have been identified in the context of minimal cartilage damage. Examples include sphingolipids, serum IL-6, CD14, CD163, MCP-1, MIP-1 beta, interferon gamma, and COMP and is being further expanded by unbiased proteomics studies [50-54].

Biomarkers of early OA were also identified in a preradiographic OA context that may predict the progression to radiographic disease.
These include serum COMP, hyaluronan, MMP-3 and -17, osteocalcin, IL-15, plasminogen activator inhibitor-1, and soluble vascular adhesion protein-1 [55-59]. Proteoglycan fragments, collagen fragments, and metalloproteinases are also detectable after injuries that predispose to $\mathrm{OA}$ such as anterior cruciate ligament (ACL) and meniscal injuries [60-65]. Persistence of such biomarkers later after injury may identify patients at higher risk of OA [66].

The combination of several biomarkers reflecting different pathological processes may better classify early OA than a single biomarker. Support for this concept was provided by a study of multiple biomarkers (urine CII neoepitope, serum hyaluronic acid, and serum pyridinoline); their integration led to higher sensitivity, specificity, and predictive ability to distinguish early OA patients from age-matched controls [67]. Complex statistical modeling is required to integrate biomarkers, imaging and clinical data to predict well-defined outcomes.

\section{Breakout session}

What should biomarkers measure? Rate of cartilage loss? Residual cartilage? Catabolism/anabolism? Inflammation? Underlying bone? Meniscal damage? Mechanistic events such as disrupted/activated pathways?

The group considered that these parameters may each have importance in different contexts. Measuring the rate of cartilage loss or other tissue parameters using biomarkers would be desirable, but challenging due to the apparent fluctuation of these parameters in individual patients over time, particularly in early OA. Biomarkers reflecting cartilage anabolism and catabolism, bone turnover, and pathogenic pathways for patient stratification by disease mechanism and activity were considered very attractive since they may aid in identifying the right therapeutic target. However, there was full consensus that these aims have not yet been fulfilled and were relevant primarily for research, and that no single marker or set of OA biomarkers have currently any utility in clinical routine.

\section{Consensus statement}

Although biomarkers may have future utility in early OA classification and prediction of progression, no individual or set of biomarkers is yet robust enough.

\section{Final session}

After the open discussion sessions, a group including the chairpersons, rapporteurs, and co-rapporteurs, met to refine a draft proposal.

There was, within this group, consensus that three classes of criteria should be used (Table):

1. Patient based questionnaires-preferably Knee Injury and Osteoarthritis Score (KOOS) that includes subdomains of pain (9 items), stiffness and other symptoms (7 items), function and daily living (short PS version 7 items), and knee-related quality of life ( 4 items). The KOOS was chosen as it contains the full set of WOMAC questions, and has additional domains making it more relevant to early $\mathrm{OA}$. The use of KOOS thus allows comparison with results from extant older cohorts, and calculation of a WOMAC score. The choice of questionnaires may be further adapted as more data, also from other cohorts, become available.

2. A second set of criteria is based on clinical examination, with two preferred clinical examination findings being joint line tenderness and crepitus. Crepitus was included as it was decided to also include patellofemoral OA and not only tibiofemoral OA. Other clinical signs such as palpable 
Table

Draft proposal for classification criteria for early OA of the knee

A. Patient-based questionnaires: Knee Injury and Osteoarthritis Outcome score: 2 out of the 4 KOOS subscales need to score "positive" ( $\leq 85 \%$ )

1. Pain (9 items, including information on pain intensity, frequency, and duration)

2. Symptoms, stiffness ( 7 items)

3. Function, daily living (short version: 7 items)

4. Knee-related quality of life (QOL: 4 items)

B. Clinical examination: at least 1 criterion needs to be present

- Joint line tenderness

- Crepitus

C. X-rays: KL grade 0-I standing, weight bearing (at least 2 projections: PA fixed flexion and skyline for patellofemoral OA)

warmth, effusion (refill test), bone tenderness, and reduced range of motion were not considered signs of early OA so were not included.

3. Standardized weight-bearing $X$-ray examination. We agreed on the radiological criteria being $\mathrm{KL} 0$ or 1 , with $\mathrm{KL}>$ 2 classified as definite OA. Two views are recommended, weight-bearing posterior-anterior fixed flexion of both knees, and a bilateral skyline (supine) view. Alignment with the OARSI recommendations was proposed [43].

It was agreed that these draft criteria need to be refined and validated in several different population-based cohorts to explore what proportion of patients defined with early OA did indeed progress to develop definite symptomatic, radiographic OA as defined by the ACR criteria.

\section{Discussion}

These draft recommendations result from consensus discussions at the first international early knee osteoarthritis workshop in Tokyo, Japan, and are intended for the international community to modify and validate.

OA remains a chronic complex disorder with modestly effective symptom relieving treatments, no approved disease modifying treatments, and with joint replacement surgery offering the most substantial relief of symptoms for severe, advanced disease. Current approaches to advance the field include stratifying patient populations into different phenotypes in order to target treatments more effectively. However, there is a growing belief that the lack of disease modifying treatments is also due to the complexity of more advanced OA disease that involves the whole joint, with degrees of irreversible damage, pain sensitization, and muscle deconditioning. It is unlikely that in an advanced disease stage targeting one pathway or one tissue will affect the disease process sufficiently in its course to be clinically relevant. It is therefore critically important to identify early OA, before these factors are all in play. This would allow us to target individuals with secondary prevention and treatment strategies more effectively with the possibility of preventing irreversible damage. We are in essence looking for the opportunity to diagnose and treat the equivalent of angina in the field of coronary artery disease to prevent myocardial infarction.

We propose that the development and validation of classification criteria for early OA would impact the field and create a positive momentum for a disease that is perceived as being both highly challenging and unlikely to be resolved by one single "wonder drug". There is a strong belief that many treatments, pharmacological and non-pharmacological, would be more effective in early $\mathrm{OA}$, by targeting single tissues or pathways before the disease becomes more complex. Importantly, classification criteria such as those proposed here do not equal diagnostic criteria, but are intended for research, to investigate new treatment strategies of early phases of the disease process, and to define valid outcomes.

We are aware that this is just the beginning of a process of producing consensus classification criteria, and that validation and further iterations of these criteria will be required. The workshop participants agreed that part of the validation process could use existing population-based cohorts for testing several constructs, as each will have its own limitations and strengths:

1. What is the specificity and sensitivity of the proposed criteria?

2. Do these criteria associate with MRI features of early knee OA?

3. Do they predict, over at least 5 years

a. the onset of ACR defined OA?

b. the onset of radiographic knee OA?

c. the need for total knee replacement?

Several community-based cohorts are available, such as the CHECK study in the Netherlands, the CAS-K(nee) population in the UK, the Osteoarthritis Initiative in the US (https://oai.epi-ucsf.org), the Chingford, Framingham, JoCo, and ROAD cohorts, that could serve to refine and validate these criteria[68-73]. MRI-related imaging and biochemical biomarkers could enter the classification criteria of early OA as they prove their utility and become more readily accessible. However, they will need high-level computational analysis to integrate them with the current criteria in models to assess both their incremental predictive ability and cost effectiveness.

We are now seeking to define and treat $\mathrm{OA}$ at an earlier stage, a stage that today may not be generally recognized as being 'real OA', so that we may intervene when there is better hope for secondary prevention by slowing or stopping further development of symptoms and tissue destruction. While doing so, we must be cognizant of the pitfalls of 'overdiagnosis' and 'overtreatment' $[74,75]$. The proposed classification criteria for early OA of the knee represent a starting point to facilitate their refinement and validation for research and to investigate new treatment strategies of early phases of the disease. The participants of the workshop were all in agreement that this is an opportunity for the field to advance the understanding and treatment of OA. Similar efforts should also follow for other joints commonly affected by OA such as the hip and hand.

\section{Role of authors}

All the authors reviewed and approved the final version of the manuscript. F.P.L., N.A., and L.S.L. wrote the first draft, coordinated the writing of the paper and brought it into its final form. As Chairmen of sessions, Sita Bierma-Zeinstra, F. Dell'Accio, V. Kraus, N. Arden, and L. S. Lohmander wrote the résumé of their 
respective sessions. K.N. and I.S. were the local organizers of the workshop and critically reviewed the paper for its content.

\section{Acknowledgments}

The authors would like to thank all participants of the first Workshop on early Osteoarthritis of the Knee, organized in November 2014 in Tokyo, Japan and hosted by the Japanese Society for Early Osteoarthritis. In particular, our thanks go to invited key speakers and contributors: C. Scanzello, H. Madry, C. De Bari, D. Hayashi, M. Englund, S. Verschueren, K. Kwoh, H. Kawaguchi, S. Wang, N. Fukui, T. Nishii, and Y. Ishibashi.

Other workshop participants: M. Lee, S. Ikegawa, Y. Zhang, A. Watanabe, M. Kloppenburg, H. Onuma, T. Mae, T. Kobayashi, F. Dell'Accio, H. Shinjo, Y. Tachibana, M. Onishi, S. Tsuji, E. Tsuda, M. Ishijima, H. Kaneko, K. Chiba, G. Omori, D. Chiba, K. Oishi, S. Takagi, N. Abe, S. Wang, N. Yoshimura, A. Yonekura, Y. Kimura, H. Koga, Y. Ikeda, K. Koizumi, E. Sasaki, H. Mera, Y. Ueda, J. Runhaar, H. Yokoi, D. Schiphof, T. Suzuki, M. Kaya, Y. Take, T. Nihsii, K. Gamada, H. Kawaguchi, T. Soejima, D. Hayashi, K. Otabe, H. Kinjo, T. Kasamatsu, T. Mine, Y. Nakagawa, K. Urano, Y. Fujishima, S. Takada, Y. Kobayashi, M. Seki, T. Nagura, M. Kimishima, T. Matsumoto, O. Lee, Y. Matsuura, T. Mizutani, J. Honda, Y. Kato, Y. Sogi, Y. Sakakibara, Y. Kii.

\section{References}

[1] Liu Q, Niu J, Huang J, Ke Y, Tang X, Wu X, et al. Knee osteoarthritis and allcause mortality: the Wuchuan Osteoarthritis Study. Osteoarthritis Cartilage 2015;23:1154-7.

[2] Hiligsmann M, Cooper C, Arden N, Boers M, Branco JC, Brandi ML, et al. Health economics in the field of osteoarthritis: an expert's consensus paper from the European Society for Clinical and Economic Aspects of Osteoporosis and Osteoarthritis (ESCEO). Semin Arthritis Rheum 2013:43:303-13 (Elsevier).

[3] Hunter DJ. Viscosupplementation for osteoarthritis of the knee. N Engl J Med 2015;372:1040-7

[4] Glyn-Jones S, Palmer A, Agricola R, Price A, Vincent T, Weinans $H$, et al. Osteoarthritis. Lancet 2015;386:376-87.

[5] Altman R, Asch E, Bloch D, Bole G, Borenstein D, Brandt K, et al. Development of criteria for the classification and reporting of osteoarthritis: classification of osteoarthritis of the knee. Arthritis Rheumatol 1986:29:1039-49.

[6] Toupin-April K, Barton J, Fraenkel L, Li L, Grandpierre V, Guillemin F, et al. Development of a draft core set of domains for measuring shared decision making in osteoarthritis: an OMERACT working group on shared decision making. J Rheumatol 2015;42:2442-7.

[7] Zhang W, Moskowitz R, Nuki G, Abramson S, Altman R, Arden N, et al. OARSI recommendations for the management of hip and knee osteoarthritis, part I: critical appraisal of existing treatment guidelines and systematic review of current research evidence. Osteoarthritis Cartilage 2007;15:981-1000.

[8] Zhang W, Doherty M, Peat G, Bierma-Zeinstra SM, Arden N, Bresnihan B, et al. EULAR evidence based recommendations for the diagnosis of knee osteoarthritis. Ann Rheum Dis 2010;69:483-9.

[9] Lohmander LS, Hellot S, Dreher D, Krantz EF, Kruger DS, Guermazi A, et al. Intraarticular sprifermin (recombinant human fibroblast growth factor 18) in knee osteoarthritis: a randomized, double-blind, placebo-controlled trial. Arthritis Rheumatol 2014;66:1820-31.

[10] Skou ST, Simonsen ME, Roos EM. Good life with arthritis in denmark (GLA: D)implementation of evidence-based care for knee and hip osteoarthritis in clinical practice. Osteoarthritis Cartilage 2014;22:S457-8.

[11] Uthman OA, van der Windt DA, Jordan JL, Dziedzic KS, Healey EL, Peat GM, et al. Exercise for lower limb osteoarthritis: systematic review incorporating trial sequential analysis and network meta-analysis. $\mathrm{Br} J$ Sports Med 2014; $48: 1579$

[12] Aggarwal R, Ringold S, Khanna D, Neogi T, Johnson SR, Miller A, et al. Distinctions between diagnostic and classification criteria? Arthritis Care Res 2015;67:891-7.

[13] Taylor WJ, Fransen J. Distinctions between diagnostic and classification criteria: comment on the article by Aggarwal et al. Arthritis Care Res 2016;68:149-50.

[14] Kellgren J, Lawrence J. Radiological assessment of osteo-arthrosis. Ann Rheum Dis $1957 \cdot 16: 494$

[15] Schiphof D, Boers M, Bierma-Zeinstra SM. Differences in descriptions of Kellgren and Lawrence grades of knee osteoarthritis. Ann Rheum Dis 2008;67:1034-6.

[16] Kastelein M, Luijsterburg PA, Belo JN, Verhaar JA, Koes BW, Bierma-Zeinstra S. Six-year course and prognosis of nontraumatic knee symptoms in adults in general practice: a prospective cohort study. Arthritis Care Res 2011:63:1287-94.
[17] Wesseling J, Welsing PM, Bierma-Zeinstra SM, Dekker J, Gorter KJ, Kloppenburg $\mathrm{M}$, et al. Impact of self-reported comorbidity on physical and mental health status in early symptomatic osteoarthritis: the CHECK (Cohort Hip and Cohort Knee) study. Rheumatology 2013;52:180-8.

[18] Case R, Thomas E, Clarke E, Peat G, et al. Prodromal symptoms in knee osteoarthritis: a nested case-control study using data from the Osteoarthritis Initiative. Osteoarthritis Cartilage 2015;23:1083-9.

[19] Hensor E, Dube B, Kingsbury SR, Tennant A, Conaghan PG. Toward a clinical definition of early osteoarthritis: onset of patient-reported knee pain begins on stairs. Data from the Osteoarthritis Initiative. Arthritis Care Res 2015;67:40-7.

[20] Wink A, Gross K, Brown C, Guermazi A, Roemer F, Niu J, et al. Varus thrust during walking and the risk of incident and worsening medial tibiofemoral MRI lesions: the Multicenter Osteoarthritis Study. Osteoarthritis Cartilage 2017:25:839-45.

[21] Mahmoudian A, van Dieën JH, Bruijn SM, Baert IA, Faber GS, Luyten FP, et al Dynamic and static knee alignment at baseline predict structural abnormalities on MRI associated with medial compartment knee osteoarthritis after 2 years. Gait Posture 2017;57:46-51.

[22] Sharma L, Chmiel JS, Almagor O, Dunlop D, Guermazi A, Bathon JM, et al Significance of preradiographic magnetic resonance imaging lesions in persons at increased risk of knee osteoarthritis. Arthritis Rheumatol 2014;66:1811-9.

[23] Schiphof D, Oei E, Hofman A, Waarsing J, Weinans H, Bierma-Zeinstra S Sensitivity and associations with pain and body weight of an MRI definition of knee osteoarthritis compared with radiographic Kellgren and Lawrence criteria: a population-based study in middle-aged females. Osteoarthritis Cartilage 2014;22:440-6.

[24] Neogi T, Guermazi A, Roemer F, Nevitt MC, Scholz J, Arendt-Nielsen L, et al. Association of joint inflammation with pain sensitization in knee osteoarthritis: The Multicenter Osteoarthritis Study. Arthritis Rheumatol 2016;68:654-61.

[25] Sharma L, Hochberg M, Nevitt M, Guermazi A, Roemer F, Crema M, et al. Knee tissue lesions and prediction of incident knee osteoarthritis over 7 years in a cohort of persons at higher risk. Osteoarthritis Cartilage 2017;25:1068-75.

[26] Guermazi A, Niu J, Hayashi D, Roemer FW, Englund M, Neogi T, et al Prevalence of abnormalities in knees detected by MRI in adults without knee osteoarthritis: population based observational study (Framingham Osteoarthritis Study). Br Med J 2012:345.

[27] Hayashi D, Felson D, Niu J, Hunter D, Roemer F, Aliabadi P, et al. Preradiographic osteoarthritic changes are highly prevalent in the medial patella and medial posterior femur in older persons: Framingham OA study. Osteoarthritis Cartilage 2014;22:76-83.

[28] Hensor EM, Dube B, Kingsbury SR, Tennant A, Conaghan PG. Toward a clinical definition of early osteoarthritis: onset of patient-reported knee pain begins on stairs. Data from the osteoarthritis initiative. Arthritis Care Res (Hoboken) 2015:67:40-7.

[29] Peat G, Duncan RC, Wood LR, Thomas E, Muller S. Clinical features of symptomatic patellofemoral joint osteoarthritis. Arthritis Res Therapy 2012; $14:$ R63.

[30] Bastick A, Verkleij S, Damen J, Wesseling J, Emans P, Bierma-Zeinstra S. Predictors for the progression of knee pain in subjects with early symptomatic knee osteoarthritis-five year results from the CHECK study. Abstracts/Osteoarthritis Cartilage 2013;21:S63-312.

[31] Kastelein M. Traumatic and Non-traumatic Knee Complaints in Genera Practice 2013.

[32] Baert IA, Staes F, Truijen S, Mahmoudian A, Noppe N, Vanderschueren G, et al Weak associations between structural changes on MRI and symptoms, function and muscle strength in relation to knee osteoarthritis. Knee Surg Sports Traumatol Arthrosc 2014;22:2013-25.

[33] Hunter D, Arden N, Conaghan P, Eckstein F, Gold G, Grainger A, et al. Definition of osteoarthritis on MRI: results of a Delphi exercise. Osteoarthritis Cartilage 2011:19:963-9.

[34] Luyten FP, Denti M, Filardo G, Kon E, Engebretsen L. Definition and classification of early osteoarthritis of the knee. Knee Surg Sports Traumatol Arthrosc 2012;20:401-6.

[35] Baert IA, Mahmoudian A, Nieuwenhuys A, Jonkers I, Staes F, Luyten FP, et al. Proprioceptive accuracy in women with early and established knee osteoarthritis and its relation to functional ability, postural control, and muscle strength. Clin Rheumatol 2013;32:1365-74.

[36] Bastick AN, Runhaar J, Belo JN, Bierma-Zeinstra SM. Prognostic factors for progression of clinical osteoarthritis of the knee: a systematic review of observational studies. Arthritis Res Therapy 2015;17:152.

[37] Bekkers JE, Inklaar M, Saris DB. Treatment selection in articular cartilage lesions of the knee. Am J Sports Med 2009;37(Suppl. 1):148S-55S.

[38] Davis MA, Ettinger WH, Neuhaus JM. Obesity and osteoarthritis of the knee: evidence from the National Health and Nutrition Examination Survey (NHANES I). Semin Arthritis Rheum 1990;20:34-41 (Elsevier).

[39] Roos EM, Lohmander LS. The Knee Injury and Osteoarthritis Outcome Score (KOOS): from joint injury to osteoarthritis. Health Qual Life Outcomes 2003;1:64.

[40] Kerkhof H, Bierma-Zeinstra S, Arden N, Metrustry S, Castano-Betancourt M, Hart D, et al. Prediction model for knee osteoarthritis incidence, including clinical, genetic and biochemical risk factors. Ann Rheum Dis 2014;73: 2116-21. 
[41] Hunter D, Nevitt M, Lynch J, Kraus VB, Katz JN, Collins JE, et al. Longitudinal validation of periarticular bone area and 3D shape as biomarkers for knee OA progression? Data from the FNIH OA Biomarkers Consortium. Ann Rheum Dis 2016;75:1607-14.

[42] Altman RD, Gold G. Atlas of individual radiographic features in osteoarthritis, revised. Osteoarthritis Cartilage 2007:15:A1-56.

[43] Hunter D, Altman R, Cicuttini F, Crema M, Duryea J, Eckstein F, et al. OARSI clinical trials recommendations: knee imaging in clinical trials in osteoarthritis. Osteoarthritis Cartilage 2015;23:698-715.

[44] Englund M, Guermazi A, Gale D, Hunter DJ, Aliabadi P, Clancy M, et al. Incidental meniscal findings on knee MRI in middle-aged and elderly persons. N Engl J Med 2008;359:1108-15.

[45] Guermazi A, Niu J, Hayashi D, Roemer FW, Englund M, Neogi T, et al. Prevalence of abnormalities in knees detected by MRI in adults without knee osteoarthritis: population based observational study (Framingham Osteoarthritis Study). Br Med J 2012;345:e5339.

[46] Hunter D, Zhang W, Conaghan P, Hirko K, Menashe L, Reichmann W, et al. Responsiveness and reliability of MRI in knee osteoarthritis: a meta-analysis of published evidence. Osteoarthritis Cartilage 2011;19:589-605.

[47] Hunter DJ, Zhang W, Conaghan PG, Hirko K, Menashe L, Reichmann WM, et al. Responsiveness and reliability of MRI in knee osteoarthritis: a meta-analysis of published evidence. Osteoarthritis Cartilage 2011;19:589-605.

[48] Katsuragi J, Sasho T, Yamaguchi S, Sato Y, Watanabe A, Akagi R, et al. Hidden osteophyte formation on plain X-ray is the predictive factor for development of knee osteoarthritis after 48 months-data from the Osteoarthritis Initiative. Osteoarthritis Cartilage 2015;23:383-90.

[49] Atukorala I, Kwoh CK, Guermazi A, Roemer F, Boudreau R, Hannon MJ, et al. Synovitis in knee osteoarthritis: a precursor of disease? Ann Rheum Dis 2016;75:390-5.

[50] Kosinska MK, Liebisch G, Lochnit G, Wilhelm J, Klein H, Kaesser U, et al. Sphingolipids in human synovial fluid-a lipidomic study. PLoS One 2014;9: e91769.

[51] Shimura Y, Kurosawa H, Sugawara Y, Tsuchiya M, Sawa M, Kaneko H, et al. The factors associated with pain severity in patients with knee osteoarthritis vary according to the radiographic disease severity: a cross-sectional study. Osteoarthritis Cartilage 2013;21:1179-84.

[52] Daghestani HN, Pieper CF, Kraus VB. Soluble macrophage biomarkers indicate inflammatory phenotypes in patients with knee osteoarthritis. Arthritis Rheumatol 2015;67:956-65.

[53] Cuellar JM, Scuderi GJ, Cuellar VG, Golish SR, Yeomans DC. Diagnostic utility of cytokine biomarkers in the evaluation of acute knee pain. J Bone Joint Surg 2009;91:2313-20.

[54] Dragomir A, Kraus V, Renner J, Luta G, Clark A, Vilim V, et al. Serum cartilage oligomeric matrix protein and clinical signs and symptoms of potential preradiographic hip and knee pathology. Osteoarthritis Cartilage 2002; 10:687-91.

[55] Ling SM, Patel DD, Garnero P, Zhan M, Vaduganathan M, Muller D, et al. Serum protein signatures detect early radiographic osteoarthritis. Osteoarthritis Cartilage 2009; 17:43-8.

[56] Golightly YM, Marshall SW, Kraus VB, Renner JB, Villaveces A, Casteel C, et al. Serum cartilage oligomeric matrix protein, hyaluronan, high-sensitivity c-reactive protein, and keratan sulfate as predictors of incident radiographic knee osteoarthritis: differences by chronic knee symptoms. Osteoarthritis Cartilage 2010;18:S62-3.

[57] Chaganti R, Kelman A, Lui L, Yao W, Javaid M, Bauer D, et al. Change in serum measurements of cartilage oligomeric matrix protein and association with the development and worsening of radiographic hip osteoarthritis. Osteoarthritis Cartilage 2008; 16:566-71.
[58] Kelman A, Lui L, Yao W, Krumme A, Nevitt A, Lane NE. Association of highe levels of serum cartilage oligomeric matrix protein and N-telopeptide crosslinks-with the development of radiographic hip osteoarthritis in elderly women. Arthritis Rheum 2006;54:236-43.

[59] Sowers M, Lachance L, Jamadar D, Hochberg MC, Hollis B, Crutchfield M, et al. The associations of bone mineral density and bone turnover markers with osteoarthritis of the hand and knee in pre- and perimenopausal women. Arthritis Rheum 1999;42:483-9.

[60] Catterall JB, Stabler TV, Flannery CR, Kraus VB. Changes in serum and synovial fluid biomarkers after acute injury (NCT00332254). Arthritis Res Therapy 2010;12:R229.

[61] Lohmander LS, Hoerrner LA, Lark MW. Metalloproteinases, tissue inhibitor, and proteoglycan fragments in knee synovial fluid in human osteoarthritis. Arthritis Rheumatol 1993;36:181-9.

[62] Chu CR, Williams AA, Coyle CH, Bowers ME. Early diagnosis to enable early treatment of pre-osteoarthritis. Arthritis Res Therapy 2012;14:212.

[63] Lohmander LS, Roos H, Dahlberg L, Hoerrner LA, Lark MW. Temporal patterns of stromelysin-1, tissue inhibitor, and proteoglycan fragments in human knee joint fluid after injury to the cruciate ligament or meniscus. J Orthop Res $1994 ; 12: 21-8$.

[64] Struglics A, Larsson S, Kumahashi N, Frobell R, Lohmander S. Changes in synovial fluid and serum cytokines and ARGS-aggrecan, and urine CTX-II and NTX-I over five years after anterior cruciate ligament rupture: an exploratory analysis in the KANON trial. Arthritis Rheumatol (Hoboken, NJ) 2015 $1816-25$.

[65] Larsson S, Struglics A, Lohmander LS, Frobell R. Surgical reconstruction of ruptured anterior cruciate ligament prolongs trauma-induced increase of inflammatory cytokines in synovial fluid: an exploratory analysis in the KANON trial. Osteoarthritis Cartilage 2017;25:1443-51.

[66] Altman R, Asch E, Bloch D, Bole G, Borenstein D, Brandt K, et al. Development of criteria for the classification and reporting of osteoarthritis. Classification of osteoarthritis of the knee. Diagnostic and Therapeutic Criteria Committee of the American Rheumatism Association. Arthritis Rheum 1986:29:1039-49.

[67] Takinami Y, Yoshimatsu S, Uchiumi T, Toyosaki-Maeda T, Morita A, Ishihara T et al. Identification of potential prognostic markers for knee osteoarthritis by serum proteomic analysis. Biomarker insights 2013;29:85-95.

[68] OsteoArthritis Initiative. Available from: https://oai.epi-ucsf.org.

[69] Wesseling J, Dekker J, Van den Berg W, Bierma-Zeinstra S, Boers M, Cats H, et al. CHECK (Cohort Hip and Cohort Knee): similarities and differences with the Osteoarthritis Initiative. Ann Rheum Dis 2009;68:1413-9.

[70] Peat G, Thomas E, Handy J, Wood L, Dziedzic K, Myers H, et al. The Knee Clinical Assessment Study-CAS (K). A prospective study of knee pain and knee osteoarthritis in the general population: baseline recruitment and retention at 18 months. BMC Musculoskelet Disord 2006;7:30.

[71] Felson DT, Naimark A, Anderson J, Kazis L, Castelli W, Meenan RF. The prevalence of knee osteoarthritis in the elderly. The Framingham Osteoarthritis Study. Arthritis Rheum 1987;30:914-8.

[72] Yoshimura N, Muraki S, Oka H, Mabuchi A, En-Yo Y, Yoshida M, et al Prevalence of knee osteoarthritis, lumbar spondylosis, and osteoporosis in Japanese men and women: the research on osteoarthritis/osteoporosis against disability study. J Bone Miner Metab 2009;27:620-8.

[73] Leyland K, Hart D, Javaid M, Judge A, Kiran A, Soni A, et al. The natural history of radiographic knee osteoarthritis: a fourteen-year population-based cohort study. Arthritis Rheum 2012;64:2243-51.

[74] Heath I. Overdiagnosis: when good intentions meet vested interests-an essay by Iona Heath. Br Med J 2013;347:f6361.

[75] Glasziou P, Moynihan R, Richards T, Godlee F. Too much medicine; too little care. British Medical Journal Publishing Group 2013;347:f4247. 